\title{
An Audio Generator System for Experimental Studies on Acoustic Biology
}

\author{
Weiming Cai, Huinong He, and Songming Zhu* \\ College of Biosystems Engineering and Food Science, Zhejiang University, \\ Hangzhou 310058, China \\ zhusmezju.edu.cn
}

\begin{abstract}
There are studies indicate that audible sound wave could have effects on propagation growth through catalyzing hearing sense organs, which would bring a series of physiological and biological chemistry reaction. The soundpressure level and frequency are important factors in propagation growth. In this study, an experimental system of audio generator was developed for investigating sound wave effect on propagation growth promotion, and an embedded development platform based on ARM+DSP+FPGA was built for the system. The DDFS (Direct Digital Frequency Synthesis) method was used to make various waveforms in the system. A feedback analysis networks was added in the system for reliable output of sound waves. Results show that the audio generator system can produce sound waves with frequency of 20 $\mathrm{Hz} 20,000 \mathrm{~Hz}$ accurately and make octave analysis of the sound in experimental environments. The new-type audio generator system will facilitate scientific researches on the field of acoustic biology.
\end{abstract}

Keywords: Audio Generator System, Acoustic Biology, ARM+DSP+FPGA, DDFS, Sound Wave.

\section{Introduction}

Audible sound, as a sort of sound waves, whose frequency is between $20 \mathrm{~Hz}$ and $20,000 \mathrm{~Hz}$, widely exists in natural environment. In recent years, there have been studies relating to sound and the growth and health of plants or animals, including human beings (Chabris, 1999; Creath and Schwartz, 2004; Collins and Foreman, 2001). It has been hypothesized that sound increases growth in plants, and some companies even use a growth system that incorporates sound to try to increase growth. Studies have been done on the use of music to improve crop yield and quality in plants such as tomato plants (Hou and Mooneyham, 1999), barley (Xiao, 1991), and vegetables (Hou et al., 2009); Hou et al. (2009) used audible sound waves to stimulate more than 50 different crops, and achieved remarkable effects. This field of science is known as acoustic biology. Up to now, why plants or animals are subjected to sound waves in order to improve crop yield, quality, and animal health is still

\footnotetext{
Corresponding author.
} 
unknown. Not much study was done in this area, as there was a lack of precise instruments to facilitate propagation growth studies in the acoustic biology field as well as a lack of proven scientific theories in this area.

Direct digital frequency synthesis (DDFS) is a method of producing an analog arbitrary waveform (Jeng et al., 2010; Chen and Chau, 2010). The operations within DDFS devices are primarily digital; it can offer faster switching between output frequencies, operation over a broader spectrum of frequencies and better frequency resolution than traditional ways (Shmelev, 2006; Shen, 2010). A practical DDFS system is often realized in hardware platform. ARM+DSP+FPGA is a new-type platform for complex system development, algorithm verification, prototype trialmanufacture, and prototype evaluation (e.g. multimedia processing system, digital communication system, and high performance instrument prototype development), and it haven't been used to design audio generator before, although it is ideal for us to perform high performance system verification and instrument development.

Therefore, in order to offer a scientific research instrument for the study of sound wave effect on propagation growth promotion in the field of acoustic biology and explore a new way in making high-performance audio generators, a new-type audio generator system based on ARM+DSP+FPGA and Technology of DDFS was proposed in the paper.

\section{Principles and Methods}

\subsection{Technology of Direct Digital Frequency Synthesis (DDFS)}

A basic Direct Digital Frequency Synthesizer consists of a phase accumulator, phaseto-amplitude converter (a Waveform Memory), a Digital-to-Analog Converter and filter as shown in Fig. 1.

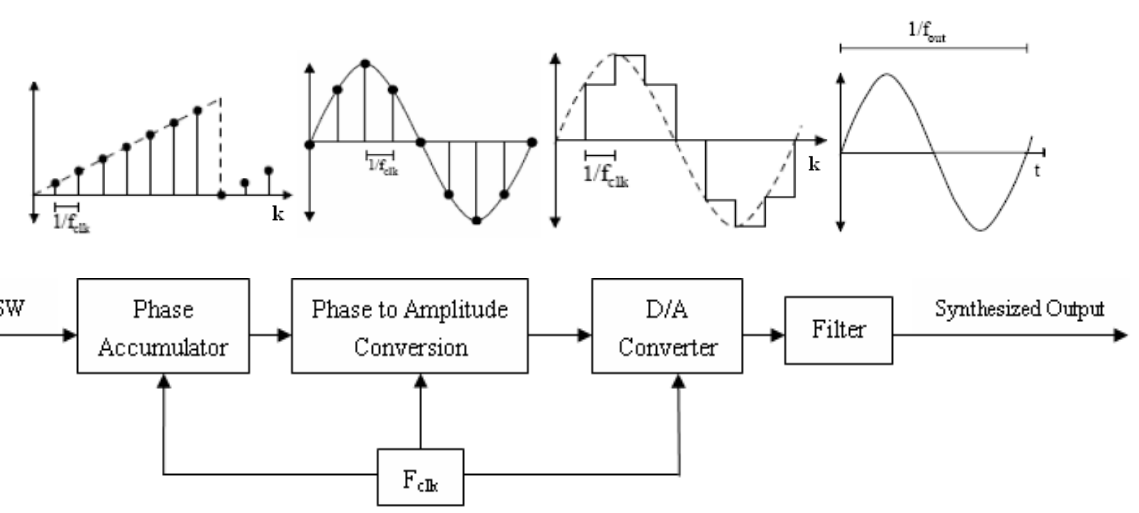

Fig. 1. Block diagram of DDFS

The frequency of synthesized output depends on three variables: a reference-clock frequency $F_{c l k}$ and a binary number programmed into the phase register (frequency 
setting word $d$ ), the length of accumulator $k$. When a sine signal is recorded in the waveform memory, the phase accumulator computes a phase (angle) address for the waveform memory, which outputs the digital value of amplitude (phase angle) to the DAC. The DAC, in turn, converts the number to a corresponding value of analog voltage or current. To generate a fixed-frequency sine wave, a constant value (the phase increment determined by the binary number $d$ ) is added to the phase accumulator with each clock cycle. When the phase increment is large, the phase accumulator will step quickly through the sine record in the waveform memory and thus generate a high frequency sine wave. When the phase increment is small, the phase accumulator will take many more steps, accordingly generating a slower waveform.

If $\mathrm{F}_{\mathrm{clk}}$ is the clock frequency, then the frequency of the output sine wave can be described as follows:

$$
F_{\text {out }}=\frac{d^{*} F_{c l k}}{2^{k}} \quad 2^{\mathrm{k}-1}>d \geq 1
$$

Where: $F_{\text {out }}$ is the output frequency of DDFS; $d$ is the frequency setting word; $n$ (in bits) is the length of the phase accumulator; $F_{c l k}$ is the system clock frequency.

Equation (1) is known as the tuning equation of DDFS. Any change to the value of $d$ results in immediate and phase-continuous changes in the output frequency. In a DDFS, no loop settling time is incurred as in the case of a PLL. As the output frequency is increased, the number of samples per cycle decreases. According to sampling theory, at least two samples per cycle are required to reconstruct the output waveform; the maximum fundamental output frequency of a DDFS is $2 F_{c l k}$. However, for practical applications, the output frequency is limited to somewhat less than that for improving the quality of the reconstructed waveform and permitting filtering on the output.

\subsection{Audio Generator Based on ARM+DSP+FPGA and DDFS}

An embedded development platform based on ARM, DSP, and FPGA was set up for the design of audio generator system. As shown in Fig. 2, the embedded development platform consists of a C6-Integra DSP+ARM Processor OMAP-L137, a Xilinx Spartan"TM-3A FPGA Platform--XC3S50A, a D/A converter CS4341, an A/D converter AK5350, sound sensor, User interface (LCD and Touch screen), Communication interface (USB and RS-232), and so on.

The OMAP-L137 C6-Integra ${ }^{\mathrm{TM}}$ (Texas Instruments Incorporated, 2010) is a processor of low-power applications based on an ARM926EJ-S and a C674x DSP core, and it enables us to quickly bring to devices featuring robust operating systems support, rich user interfaces, and high processing performance through the maximum flexibility of fully integrated mixed processor solutions. The ARM926EJ-S is a 32-bit RISC processor core that performs 32-bit or 16-bit instructions and processes 32-bit, 16-bit, or 8-bit data, and the core uses pipelining so that all parts of the processor and 


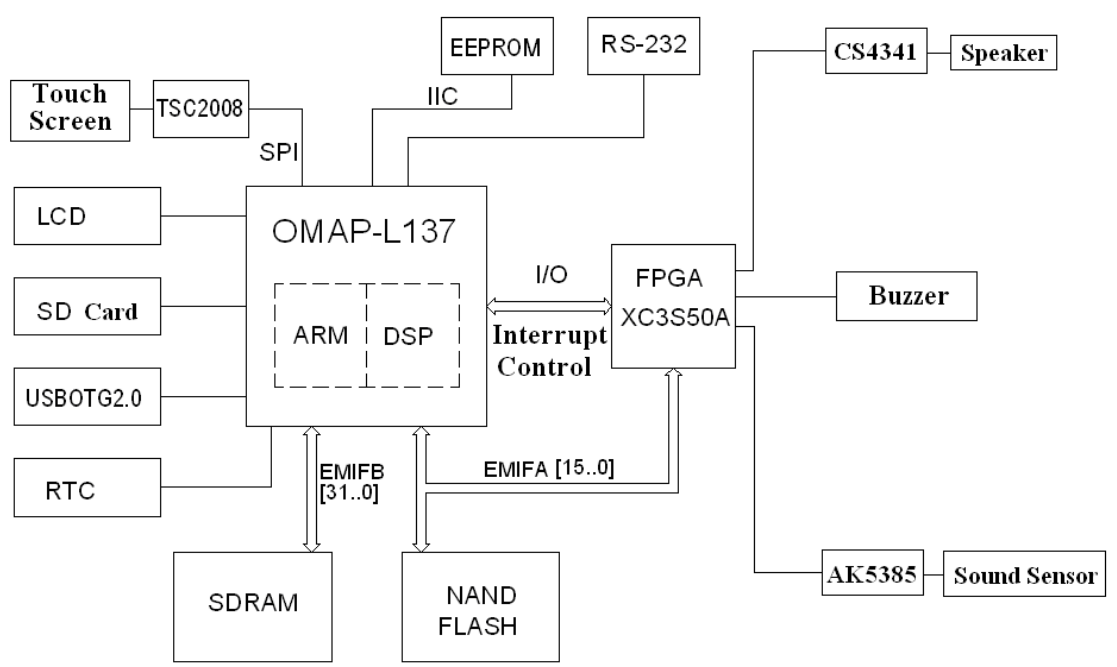

Fig. 2. Schematic diagram of embedded platform for audio generator design

memory system can operate continuously. The OMAP-L137 DSP core uses a twolevel cache-based architecture: the Level 1 program cache (L1P) is a $32 \mathrm{~KB}$ direct mapped cache and the Level 1 data cache (L1D) is a 32KB 2-way set-associative cache; the Level 2 program cache (L2P) consists of a $256 \mathrm{~KB}$ memory space that is shared between program and data space. L2 memory can be configured as mapped memory, cache, or combinations of the two. Although the DSP L2 is accessible by ARM and other hosts in the system, an additional 128KB RAM shared memory is available for use by other hosts without affecting DSP performance. In our design, the functions such as User interface, frequency setting control for the DDFS system, feedback analysis for output sound waves are mainly depend on the OMAP-L137 processor, which is one of the cores in the design of audio generator.

The Spartan-3A FPGA XC3S50A (Xilinx, 2007) is one of programmable chips with $1.4 \mathrm{M}$ system gates, and $502 \mathrm{I} / \mathrm{Os}$, with density migration. The phase accumulator and phase to amplitude conversion of DDFS is realized through programming in the FPGA; the FPGA also was used to the data communications and external state control in our design.

The CS4341 is a complete stereo digital-to-analog system including digital interpolation, fourth-order Delta-Sigma digital-to-analog conversion, digital deemphasis and switched capacitor analog filtering. The advantages of this architecture include: ideal differential linearity, no distortion mechanisms due to resistor matching errors, no linearity drift over time and temperature and a high tolerance to clock jitter. The CS4341 can accept data at audio sample rates from $4 \mathrm{kHz}$ to $100 \mathrm{kHz}$. There is a filter in the inner of CS4341. So it is not necessary for us to design additional filter for the DDFS module.

The AK5385A is a 24 bit, $192 \mathrm{kHz}$ sampling 2ch A/D converter for high-end audio system. The modulator in the AK5385A uses the Enhanced Dual Bit architecture and the AK5385A realizes high accuracy and low cost. The AK5385A performs $114 \mathrm{~dB}$ 
dynamic range, which can meet the dynamic range from $0 \mathrm{~dB}$ to $110 \mathrm{~dB}$ of our design requirement. By using a sound pressure sensor, the $\mathrm{A} / \mathrm{D}$ converter and programming in the OMAP-L137 (the sound analysis are mainly made by the C674x DSP core), the feedback analysis network can be accomplished.

\section{Results and Discussion}

In order to see whether the accomplished audio generator system can satisfy the demand of studies of sound wave effect on propagation growth promotion, a series of tests were conducted.

Fig. 3 is the time domain signal graph of sound wave detected by the feedback block of the accomplished audio generator system when a sine wave with frequency of $1000 \mathrm{~Hz}$ was inputted from the User interface of the generator. The generator system can produce sound wave signals with frequencies range from $20 \mathrm{~Hz}$ to $20,000 \mathrm{~Hz}$ for the speaker.

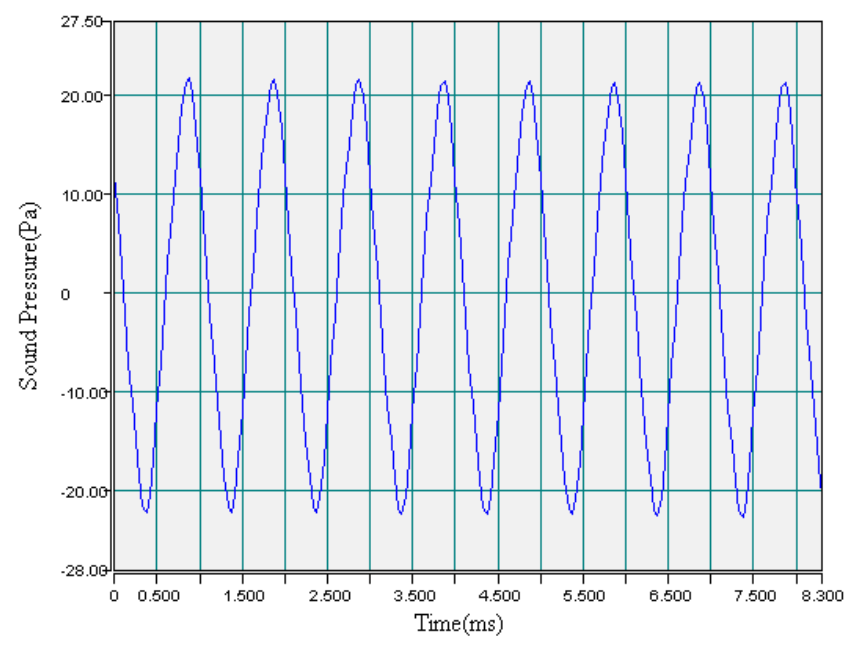

Fig. 3. Time domain signal graph of $1000 \mathrm{~Hz}$ sound wave

Natural sound such as cricket sound may have effect on propagation growth according to many studies (Chabris, 1999; Creath and Schwartz, 2004; Collins and Foreman, 2001; Hou et al., 2009). The generator can simulate the cricket or other insects' or birds' sound for the studies and the sound frequency can also be analyzed by the generator. Fig. 4 is the time domain signal graph of sound wave detected by the feedback block of the accomplished audio generator when cricket sound wave was inputted from the User interface of the generator.

Sound-level measurements offer a conventional way to measure sound but do not contain frequency information, making it difficult to compare different sounds effect in our research. Octave analysis filters the signal and measures the energy at the 


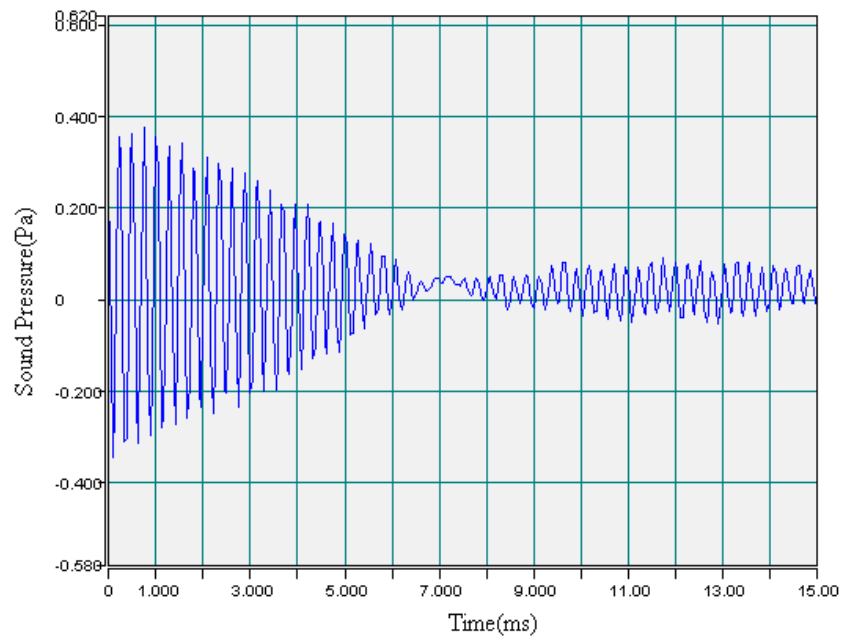

Fig. 4. Time domain signal graph of cricket sound wave

output to provide useful frequency information. With fractional-octave analysis, we can select a frequency resolution that is well adapted to the signal of interest. In a word, Octave analysis is a valuable tool for visual inspection and comparison during our researches. The new-type audio generator system offer octave analysis of output sound both for the output regulation and frequency information.

Some octave analysis was made by the generator system when the test work was conducted. The results can be seen in Figs. 5-8.

Fig. 5 shows the octave analysis chart of background noise in our testing environment. The background noise is also a factor in the research on propagation growth. So it will give us deeper understanding on the grow environment and help to explore growth promotion secrets to analysis background noise.

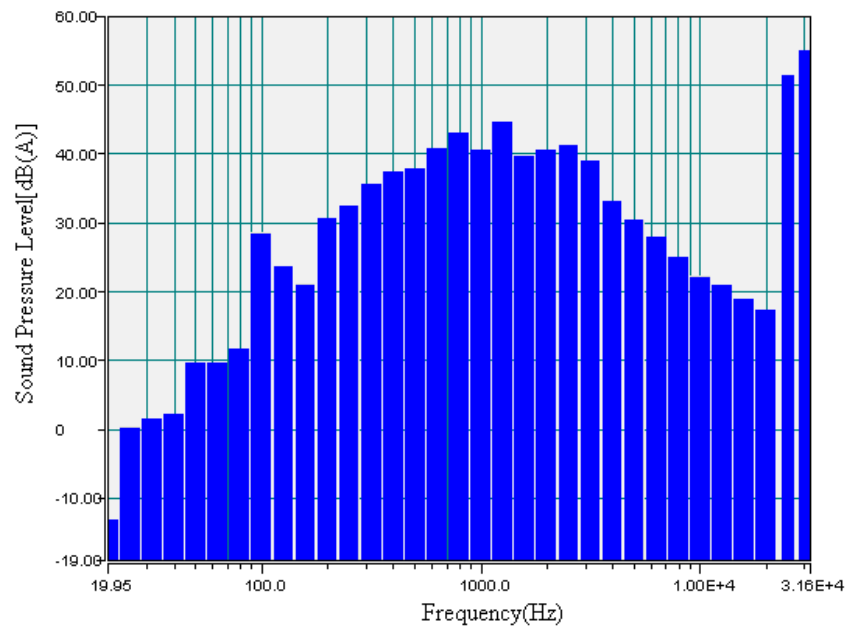

Fig. 5. The octave analysis chart of background noise 
Fig. 6 is the octave analysis chart of cricket sound. Through the octave analysis on cricket sound, we can find that frequencies around $4000 \mathrm{~Hz}$ are main frequency components in cricket sound. This offers us a scientific way in study of insects' sound effect on propagation growth.

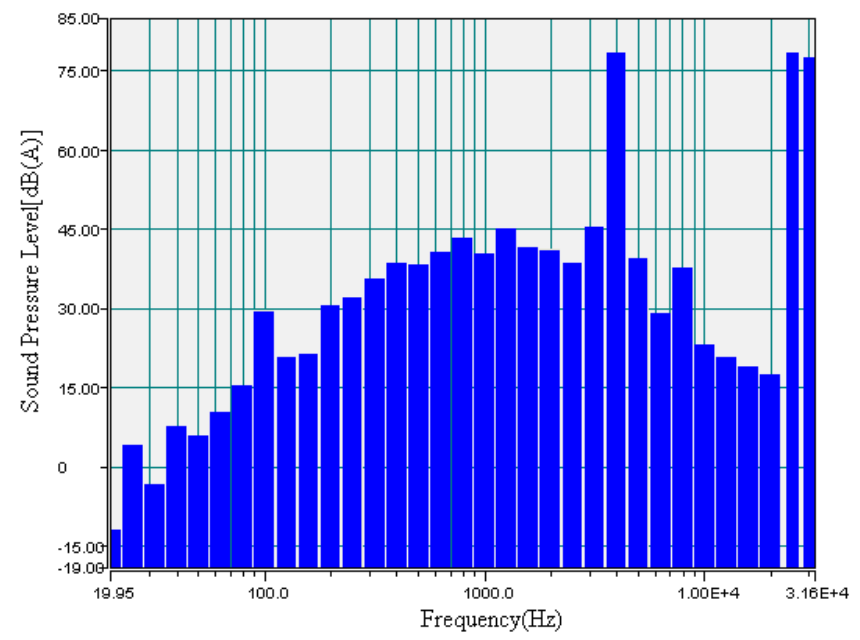

Fig. 6. The octave analysis chart of cricket sound

Fig. 7 shows the octave analysis chart of thrush sound. Through the octave analysis on thrush sound, it can be found that frequencies around $2000 \mathrm{~Hz}$ are main frequency components in thrush sound. This offers us a scientific way in study of birds' sound effect on propagation growth.

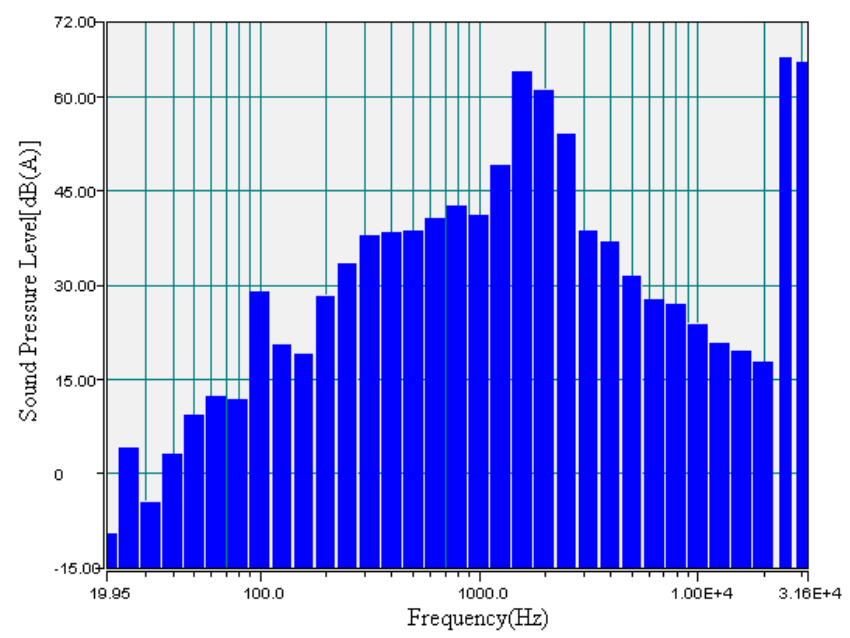

Fig. 7. The octave analysis chart of thrush sound 
Fig. 8 is the octave analysis chart of a slice of Mozart music (Allegro Molto). Through the octave analysis on Mozart music, it can be found that frequencies around $1500 \mathrm{~Hz}$ are main frequency components in the Mozart music. This offers us a scientific way in study of music effect on propagation growth.

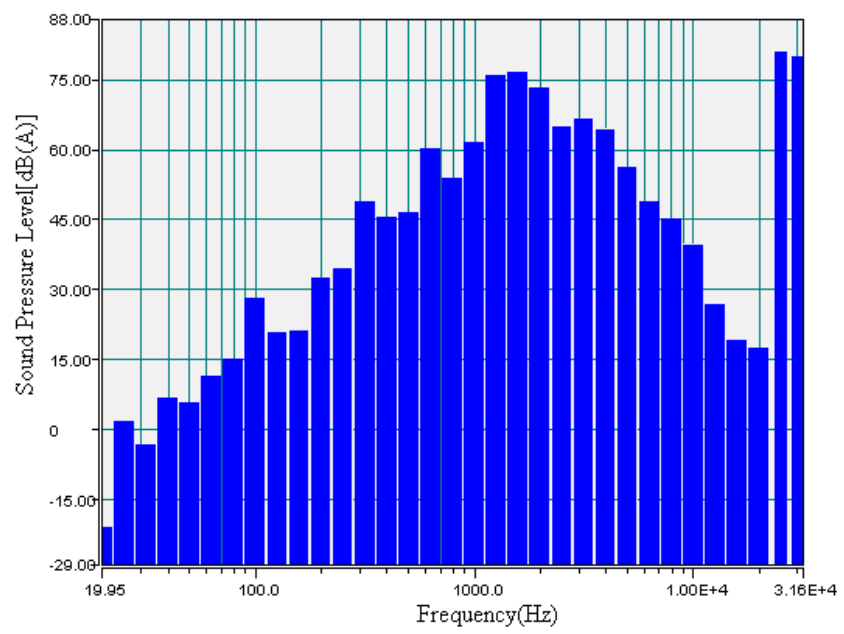

Fig. 8. The octave analysis chart of Mozart music (Allegro Molto)

From the test results described above, It can be found that the new-type audio generator system designed in the study not only can produce varies kind of sound wave but also can monitor the sound output and make analysis of the sound wave in the experimental environment. When we make researches on sound wave effects of propagation growth promotion, it is very important to load a specific sound exactly and understand the environment sound characteristic. Therefore, the audio generator designed in the study will be a useful tool to studies in the field of acoustic biology.

\section{Conclusions}

A new-type audio generator system was designed for the study of sound wave effect on propagation growth promotion in the acoustic biology field. The generator system can produce varies kind of sound required in the study of acoustic biology, and it can also do octave analysis of the sound wave in the experiment environments for acoustic biology researchers.

Unlike most of traditional audio generator systems, the generator designed in this study is based on a new embedded development platform with the ARM+DSP+FPGA core and DDFS technology, which enable us to make feedback analysis networks for the output sound and generate arbitrary waveforms. 
Acknowledgments. Research grant support from the Key Laboratory of EcoAgricultural Environmental Engineering and Intelligent Equipment of China Agricultural Ministry (Grant Number: 588040*172210214[2]) is acknowledged.

\section{References}

1. Chabris, C.: Prelude or requiem for the "Mozart effect"? Nature 400, 826-827 (1999)

2. Collins, M., Foreman, J.: The effect of sound on the growth of plants. Canadian Acoustics 29, 3-8 (2001)

3. Creath, K., Schwartz, G.E.: Measuring Effects of Music, Noise, and Healing Energy Using a Seed Germination Bioassay. The Journal of Alternative and Complementary Medicine 10(1), 113-122 (2004)

4. Jeong, M.J., Shim, C.K., Lee, J.O.: Plant genes responses to frequency-specific sound signals. Molecular Breeding 21(2), 217-226 (2008)

5. Hou, T., Mooneyham, R.: Applied studies of Plant Meridien System: I. The Effect of Agriwave Technology on Yield and Quality of Tomato. American Journal of Chinese Medicine 27, 1-10 (1999)

6. Xiao, H.: Vegetables and music. Pictorial Science 6, 36 (1991)

7. Hou, T., Luan, J., Wang, J., Li, M.: Experimental evidence of a plant meridien system III: The Sound Chracteristics of Phylodendron (Alocasia) and the Effects of Acupuncture on those Properties. American Journal of Chinese Medicine 22, 205-214 (1994)

8. Hou, T., Li, B., Guanghui, T., et al.: Application of acoustic frequency technology to protected vegetable production. Transactions of the Chinese Society of Agricultural Engineering 25(2), 156-159 (2009) (in Chinese)

9. Jeng, S., Lin, H., Wu, C.: High-Performance DDFS Design Using the Equi-Section Division Method. IEEE Transaction on Ultrasonics Ferroelectrics and Frequency Control 57(12), 2616-2626 (2010)

10. Chen, Y., Chau, Y.: A Direct Digital Frequency Synthesizer Based on a New Form of Polynomial Approximations. IEEE Transactions on Consumer Electronics 56(2), 436-440 (2010)

11. Shmelev, O.: A two-channel multi-tone computer audio-frequency generator. Measurement Techniques 49(1), 57-58 (2006)

12. Shen, J.: Picosecond fast arbitrary waveform pulse generator. Optical Fiber \& Electric Cable and Their Applications 1(2), 11-14 (2010) (in Chinese)

13. Yi, S.: A direct digital frequency synthesizer based on ROM free algorithm. AeuInternational Journal of Electronics and Communications 64(11), 1068-1072 (2010)

14. Texas Instruments Incorporated. OMAP-L137 C6-Integra DSP+ARM Processor (Rev. D) (2010),

http: / / focus.ti.com/docs/prod/folders/print/omap-1137.html

15. Xilinx. Spartan-3AN FPGA Family Data Sheet (2007),

http: //www.xilinx. comport/documentation/

data_sheets/ds529.pdf 